\title{
A Review on Face Recognition Attendance System
}

\author{
Shubhi Shriwastav \\ M. Tech Scholar, \\ Department of Computer Science \& Engineering, \\ SIRTS, RGPV Bhopal
}

\author{
Dinesh Chandra Jain \\ Prof. Department of Computer Science \& \\ Engineering, \\ SIRTS, RGPV Bhopal
}

\begin{abstract}
In this paper, we advise a method that takes the presence of students for classroom lecture. Our method takes the presence automatically using face recognition. However, it is complicated to calculate approximately the attendance exactly using each result of face detection independently because the face recognition rate is not sufficiently high. In this paper, we propose a process for estimate the presence precisely using all the results of face recognition obtained by Continuous inspection. Continuous inspection improves the performance for the estimation of the presence we Constructed the lecture presence system based on face recognition, and applied the system to classroom lecture. Face recognition technology has widely concerned concentration due to its huge application value and market potential, such as face recognition and video observation system. Real-time face detection not only is one part of the automatic face recognition system but also is increasing an independent research subject. So, there are lots of approaches to solve face detection. This paper describes the expansion of a student attendance system based on face recognition and authentication technology. MATLAB outline is used for implementation. The existing conventional presence system requires students to manually sign the sheet every time
\end{abstract}

\section{Keywords}

Face recognition,

\section{INTRODUCTION}

Face detection equipment has widely attracted attention due to its enormous application value and market potential, such as face detection and video surveillance system. A new approach in automatic attendance management systems extended with computer vision algorithms. A real time faces detections algorithms which automatically detects and registers student's presence on a lecture. The system represents a supplemental device for instructors, combine algorithms used in machine learn with adaptive methods used to track facial changes through a longer period of time. This new structure aims to be less time consuming than conventional methods, at the same time being nonintrusive and not interfere with the normal teaching process. The tool promise to offer correct outcome and a more in depth treatment system which shows student movement and presence in a classroom. A new automatic attendance management marking system, without any interference with the regular teaching process. The system can be used also during exam sessions or other teaching activities where attendance is obligatory. This system eliminates classical student identification such as calling student names, or checking respective identification cards, which can not only interfere with the teaching process, but also can be stressful for students during exam sessions. Normally sheet is passed around the classroom as the professor is conducting the lecture. For illustration, lecturers with a large class may find the difficulty of having the attendance sheet being passed around the class and the manual signing of presence by students are burdensome and most student are not concentrating on teaching [1]. In terms of analysis, the professor also has to perform manual calculation to obtain the students' attendance percentage, which normally consume a lot of time. As a consequence of so as to, professors can no longer map out the students overall record throughout the particular time. Apart from that, a professor also has restricted access to the single-copy record.

Some students may accidentally sign a further student's name. The first case leads to a student not there but their name was, while the latter lead to a false presence record. Another concern of having the record in a durable copy form is that a lecturer may lose the sheet [2]. Sometimes storage space of sheets needs cupboard or lots of space. The limitations imposed by the conventional system, we propose a solution in the form of a presence tracking method.

Though the record streaming service of lecture collection is readily accessible in many systems, student has little chance to view the speech in this service because speech content is not summarized. If the presence of a student of classroom teach is fix to the video streaming service, it is likely to present the video of the time when he was missing. It is important to take the presence of the students in the classroom without human intervention.ID tag or other identifications such the proof of login/out in most e-Learning systems are not sufficient because it does not represent student's situation in face-to-face classroom. It is also difficult to grasp the contexts by the data of a single moment. The attendance using face recognition based on continuous observation. In this paper, our purpose is to obtain the presence, position and images of students' face, which are useful information in the classroom lecture.

Fig. 1 shows that how GUI works for input image processing. Overview of capturing image from outside world and Save in database. If picture is not captured correctly then it will gives error otherwise it display output message of matching face.

Maintaining the attendance is very important in all the institutes for checking the performance of employees. Fig. 2 shows that steps of identifying face process. 


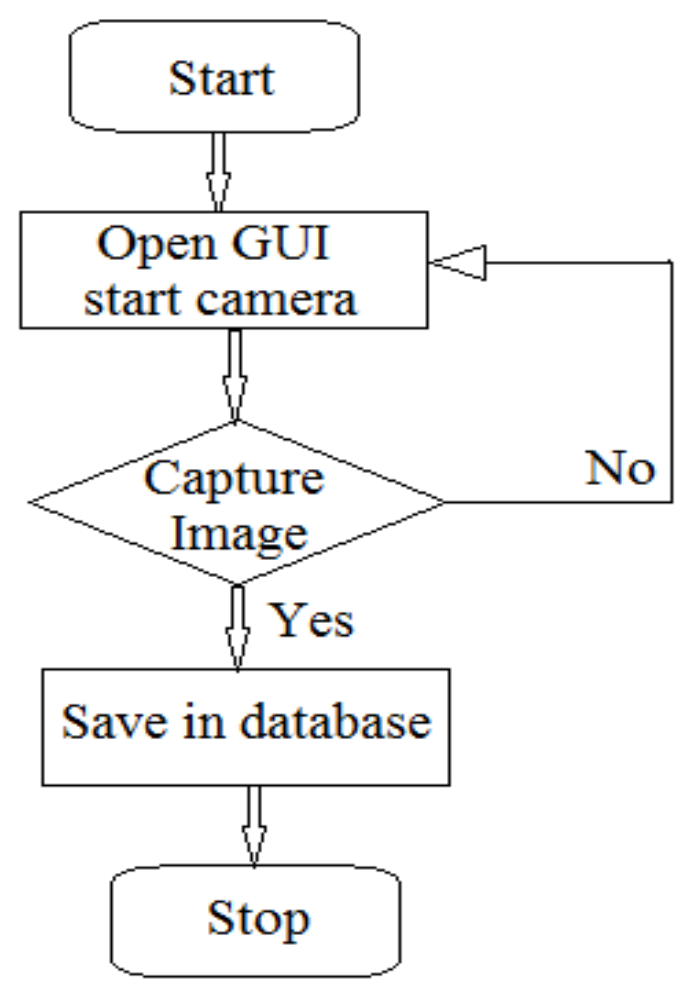

Fig. 1: GUI Input Image Flowchart

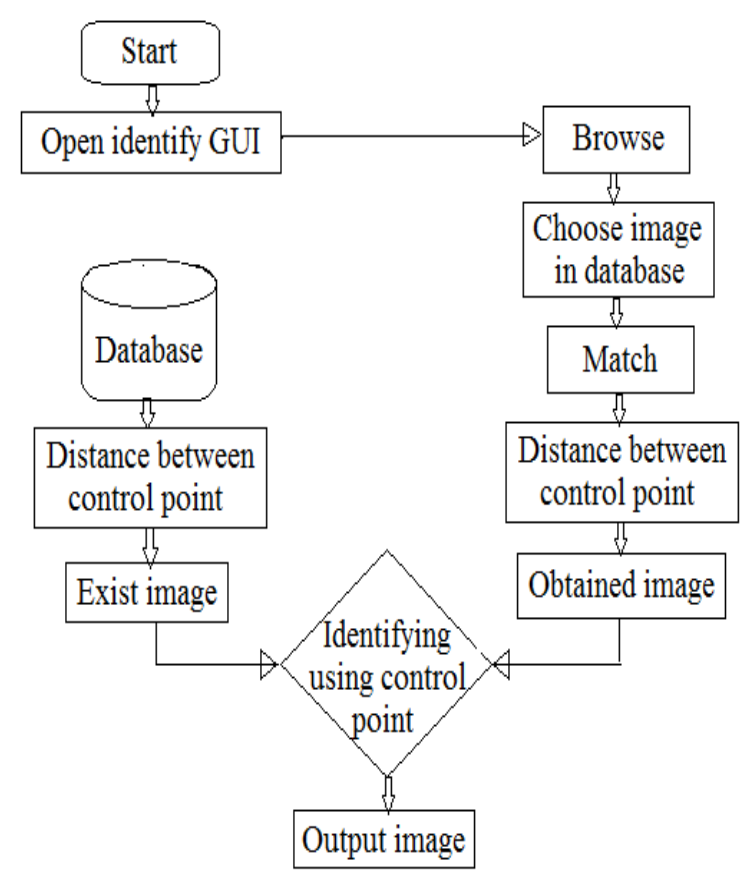

Fig. 2: Flowchart of the GUI of Identify Face Process

\subsection{Face detection and recognition}

Face detection and recognition section detect face from the image capture by the camera, and the image of the face is crop and store. The element recognizes the images of student's face, which have been register manually with their names and ID code in the record. Face recognition data and face identification data are verification into the record.

Automatic face recognition (AFR) technology has seen remarkable improvement in presentation over the past years, and such systems are now widely used for safety and marketable applications. An automatic system for human face recognition in a real time environment for a university to mark the attendance of their employees.

\section{LITERATURE SURVEY}

K.Senthamil Selvi et.al. [1] "Face recognition based Attendance marking system". In this projected work, sort to find the attendance, positions and face descriptions in classroom lecture, we projected the presence administration system based on face detection in the classroom lecture. The system estimates the presence and the location of each student by continuous inspection and footage. The result of our beginning experiment shows continuous inspection improved the performance for estimation of the attendance.

Mr.C.S.Patil et.al. [2] "Student Attendance Recording System Using Face Recognition with GSM Based", Student footage system using face validation was considered and implemented. It was tested with dissimilar face images. This idea is working properly with different panel. All windows are running separately and equivalent. If appreciation is to participate as a viable biometric for validation, then a further order of improvement in detection score is necessary. Under controlled condition, when lighting and pose can be controlled, this may be possible. It is more likely, that future improvement will rely on making better use of video knowledge and employing fully 3D face models.

Muhammad Fuzail et.al. [3] "Face Detection System for Attendance of Class' Students", An regular attendance supervision system is a essential tool for any LMS. Most of the existing system are time consuming and necessitate for a semi instruction manual work from the instructor or students. This approach aim to explain the issues by integrates face detection in the procedure. Even though this method still lacks the capability to identify each student in attendance on class, there is still much more room for enhancement. Since we implement a modular approach we can get better different module until we reach an acceptable detection and identification rate. Another issue that has to be taken in consideration in the opportunity is a process to ensure users privacy. Whenever you like a representation is stored on servers, it must be impossible for a person to use that image.

Mathana Gopala et. al. [4] "Implementation of Automated Attendance System using Face Recognition", automated presence System has been envision for the purpose of falling the errors that occur in the conventional (manual) attendance taking system. The aim is to computerize and make a system that is useful to the institute such as an organization. The efficient and exact method of attendance in the office atmosphere that can reinstate the old manual methods. This technique is secure enough, reliable and available for use. No need for dedicated hardware for installing the system in the office. It can be constructed using a camera and computer.

S. B. Dabhade et. al. [5] "Face Recognition using Principle Component Analysis and Linear Discriminate Analysis Comparative Study" recognition Rate some time enlarged sometime reduce, sometime stable. We have done various research like particular image for enrolment and particular image for testing, then we had keep the enrolment image as constant and change testing images such as two, three, four, up to nine. For taking the consequence we had used three databases ORL Database, KVKR-Face Database and IITIndian Database. 


\section{METHOD}

Biometric is the mainly secure and suitable authentication instrument. It cannot be rented, stolen, or forgotten and forging one is basically impossible. Biometrics calculates individual's exclusive physical or behavioral characteristics to make out or confirm their identity. Common physical biometrics includes fingerprints, hand or palm geometry, retina, iris, and facial characteristics.

Biometric is programmed methods of identify a person or verifying the characteristics of a person based on a physiological or behavioral point. Examples of physiological character include hand or finger images, facial character. Behavioral characters are qualities that are learned or acquire. Dynamic signature authentication, speaker verification and keystroke dynamics are examples of behavioral character. Biometric confirmation requires comparing a registered or enrolled biometric sample beside a newly captured biometric sample for example, a fingerprint capture during a login. During enrollment a sample of the biometric attribute is captured, processed by a computer, and stored for later comparison.[1].

\subsection{Security Parameters of Biometrics Unique}

The different biometrics systems have been developed around unique characteristics of individuals. The probability of 2 people giving out the same biometric data is virtually zero.

Cannot be Shared: Since a biometric belonging is an intrinsic property of an individual, it is particularly difficult to duplicate or share (you cannot give a copy of your appearance or your hand to someone!).

Cannot be copied: Biometric characters are nearly unworkable to fake or send-up, especially with new technologies ensure that the biometric person identified is from a live one.

Cannot be Lost: A biometric belongings of an individual can be lost only in case of serious accident.

Working theory of Biometric Technologies-

Biometric technology takes advantage of upon unique, permanent, and scan able human characteristics. A unique feature is one that no further person shares. This feature should also remain the same over time, and be consistently collectable by means of a sensor. As much as achievable, biometric technologies focus upon these types of person behavior.

All biometric devices take an amount of measurements from a person then digitally method the result of these measurements and save this illustration of the individual's behavior into a template. Templates are then store in a record associated with the piece of equipment or in a smartcard given to the person. This is called enrollment.

At their most basic level, biometric technology are pattern approval systems that use either image acquisition procedure, such as scanners or cameras in the case of fingerprint or iris detection technologies, or sound or movement acquisition procedure, such as microphones or platens in the case of voice detection or signature detection technologies, to collect the biometric patterns or characteristics.[5]

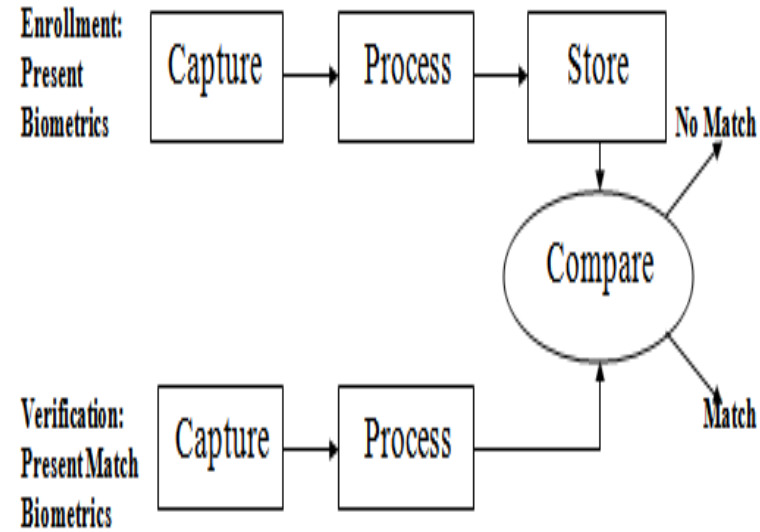

Fig.3: Generic biometric processes

\subsection{Biometric Techniques}

Biometrics consists of method for individually recognizing humans based upon one or further intrinsic physical or behavioral qualities. In computer science, in exacting, biometrics is used as a form of personality access management and access control. It is also used to identify persons in groups that are under observation. Biometric characteristics can be divided in two main classes Physiological are interrelated to the shape of the body. Examples include, but are not limited to fingerprint, face appreciation, DNA, Palm print, hand geometry, iris appreciation, which has largely replace retina, and odor/scent. Behavioral are related to the performance of a person.

Examples comprise, but are not limited to typing regularity, step, and voice. Some researchers have coined the term performance metrics for this class of biometrics.

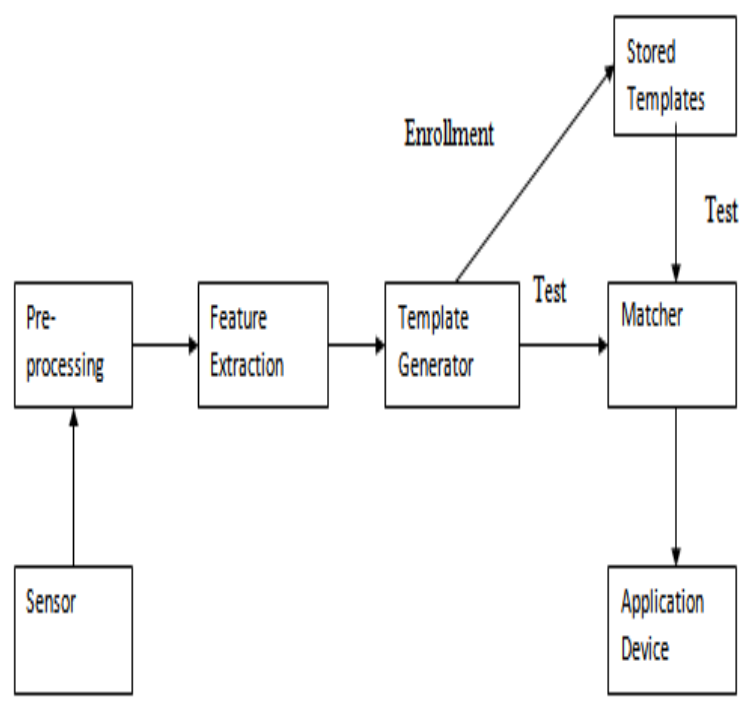

Fig.4: Biometric Technique

\section{CONCLUSION}

Student video recording method using face authentication was designed and implemented. It was tested with dissimilar face images. This idea is working suitably with different panel. All windows are running separately and parallel. If recognition is to contend as a practicable biometric for authentication, then a further order of development in detection score is required. This system provides some extra imminent into the field of 
image dealing out and contributes to the improvement of the field. Face as a biometric modality is widely acceptable for the general public, and face detection tools is able to meet the accurateness demands of a wide range of applications. Also we realize that this project saves time, power and therefore atmosphere. Charge of printing card is also reduced. In addition, we found that our database was preventive. The MATLAB code was developed and it met the design criteria and solves the problem. Future work will be paying attention on verifying the algorithm performance against general images and studying the required modification to make the algorithm strong with any image. These thoughts will be implemented in future.

\section{REFERENCES}

[1] K.Senthamil Selvi, P.Chitrakala, A.Antony Jenitha, "Face recognition based Attendance marking system" IJCSMC, Vol. 3, Issue. 2, February 2014, pg.337 - 342

[2] Mr.C.S.Patil, Mr.R.R.Karhe, Mr.M.D.Jain, "Student Attendance Recording System Using Face Recognition with GSM Based" International Journal of Research in Advent Technology, Vol.2, No.8, August 2014 E-ISSN: 2321-9637

[3] Muhammad Fuzail, Hafiz Muhammad Fahad Nouman, Muhammad Omer Mushtaq, Binish Raza, Awais Tayyab, Muhammad Waqas Talib, "Face Detection System for Attendance of Class' Students" International journal of multidisciplinary sciences and engineering, vol. 5, no. 4, April 2014

[4] Mathana Gopala Krishnan, Balaji, Shyam Babu, "Implementation of Automated Attendance System using Face Recognition" International Journal of Scientific \& Engineering Research, Volume 6, Issue 3, March-2015

[5] S. B. Dabhade, Y. S. Rode, M. M. Kazi, R. R. Manza and K. V. Kale, "Face Recognition using Principle Component Analysis and Linear Discriminant Analysis
Comparative Study" Responsibility of contents of the paper rest upon the author and not upon the publisher, Programme chair and AEMDS 2013

[6] Gatsheni, B.N., R.B. Kuriakose, and F. Aghdasi.Automating a student class attendance register using radio frequency identification in South Africa. in Mechatronics, ICM2007 4th IEEE International Conference on. 2007.

[7] Tokiwa, Y., K. Nonobe, and M. Iwatsuki. Web based tools to sustain the motivation of students indistance education. in Frontiers in Education Conference, 2009. FIE '09. 39th IEEE. 2009.

[8] Chuo-Ling Chang, Edward Li, Zhifei Wen, Rendering Novel Views of faces Using Disparity Estimation. Stanford EE368 Spring 2000/2001 Final Project.

[9] M.A. Turk, A.P. Pentland. "Face Recognition Using Eigenfaces," IEEE Conference on Computer Vision and Pattern Recognition, pp.586--591, 1991.

[10] X. Li and S. Areibi, "A Hardware/Software Codesign Approach for Face Recognition," Proc. 16th International Conference on Microelectronics, Tunis, Tunisia, Dec 2004.

[11] Visar Shehu, Agni Dika, "Using Real Time Computer Vision Algorithms in Automatic Attendance Management Systems" ITI 2010 32nd Int. Conf. on Information Technology Interfaces, June 21-24, 2010, Cavtat, Croatia.

[12] PAN Xiang, "Research and Implementation of Access Control System based on RFID and FNNFace, 2012 International Conference on Intelligent Systems Design and Engineering Application $12 \quad 2012 \quad 2012.400$ Recognition”, 978-0-7695-4608-7/11 \$26.00 (C) 2011 IEEE 